\title{
On Path Selection and Rate Allocation for Video in Wireless Mesh Networks
}

\author{
Sastry Kompella, Member, IEEE, Shiwen Mao, Member, IEEE, Y. Thomas Hou, Senior Member, IEEE, and \\ Hanif D. Sherali
}

\begin{abstract}
Multi-path transport is an important mechanism for supporting video communications in multihop wireless networks. In this paper, we investigate the joint problem of optimal path selection and rate allocation for multiple video sessions in a wireless mesh network. We present a mathematical formulation to optimize the application level performance (i.e., video distortion) in the context of path selection and rate allocation. For this complex optimization problem, we propose a branch-and-bound based solution procedure, embedded with the Reformulation-Linearization Technique (RLT) that can produce $(1-\epsilon)$-optimal solutions for any small $\epsilon$. This result is significant as it not only provides theoretical understanding of this problem, but also offers a performance benchmark for any future proposed distributed algorithm and protocol for this problem. Simulation results are also provided to demonstrate the efficacy of the solution procedure.
\end{abstract}

Index Terms-Cross-layer design, optimization, path selection, rate allocation, video communications, wireless mesh network.

\section{INTRODUCTION}

$\mathbf{R}$ ECENTLY, there has been a growing interest in supporting video communications in multihop wireless networks. Under this setting, multi-path transport is considered as one of the most important mechanisms. Indeed, there are a number of significant advantages in the use of multi-path for video communications, such as load balancing, potentially higher video bit rate, and improved error resilience, among others.

Multi-path routing, which exploits path diversity in a mesh topology, has been an active research area over the years. Various algorithms have been proposed for problems such as computing $k$-shortest paths [8], node- or link-disjoint paths [15], or braided multiple paths [13]. In multimedia applications, path diversity has been exploited to mitigate link failures and improve the reliability of streaming video [1], [4], [16]. While algorithms for finding a set of paths between a source and destination are not difficult to develop, it remains a challenging task to select

Manuscript received November 10, 2005; revised December 12, 2006 and November 09, 2007; approved by IEEE/ACM TRANSACTIONS ON NETWORKING Editor M. Krunz. First published July 02, 2008; current version published February 19, 2009. This work was supported by the Office of Naval Research under Grants N00014-03-1-0521 and N00014-05-1-0481, and by the National Science Foundation under Grants DGE-9987586, CNS-0347390 and DMI-0552676.

S. Kompella was with Virginia Tech, Blacksburg, VA 24061 USA. He is now with the Information Technology Division, U.S. Naval Research Laboratory, Washington, DC 20375 USA.

S. Mao was with Virginia Tech, Blacksburg, VA 24061 USA. He is now with the Department of Electrical and Computer Engineering, Auburn University, Auburn, AL 36849 USA (e-mail: smao@ieee.org).

Y. T. Hou is with the Bradley Department of Electrical and Computer Engineering, Virginia Tech, Blacksburg, VA 24061 USA (e-mail: thou@ vt.edu).

H. D. Sherali is with the Grado Department of Industrial and Systems Engineering, Virginia Tech, Blacksburg, VA 24061 USA (e-mail: hanifs@ vt.edu).

Digital Object Identifier 10.1109/TNET.2008.925942 an optimal subset of paths to transport video and maximizes its quality. Further, for video communications, it is also necessary to compute the optimum video encoding rate before deciding how to allocate this source rate among a given set of paths to the destination.

This paper aims to address this problem by investigating the following questions: i) at what rate should the video be encoded at the source; and ii) how to split the video source rate among a set of given paths so that the reconstructed video quality at the receiver is maximized. Instead of considering only a single user session in a network, we study multiple concurrent sessions in a network that share the same network resource. Due to the interactions among these competing sessions, the video encoding rates for all the video sessions are dependent upon each other. With this relationship, it is not hard to see that the joint problem of path selection and rate allocation in a multi-session wireless mesh network is mathematically challenging.

In this paper, we study this important problem. Our goal is to develop some theoretical results (i.e., solution with provable performance guarantee) instead of heuristics. We first formulate the joint path selection and rate allocation problem into a mathematical programming problem. We use application layer performance metric (e.g., video distortion) as our objective function and model it as a function of path selection and rate allocation. For video applications, each packet from its source is also associated with a decoding deadline. This decoding deadline sets a maximum delay bound for a packet to be successfully delivered to the receiver in order to contribute to the decoding process. In our formulation, delays on each link due to interactions among competing video sessions are considered, and the end-to-end delay distribution is derived using the Chernoff bound approximation [5]. We show that such modeling enables a tractable cross-layer formulation.

Not surprisingly, our cross-layer formulation falls into a nonconvex optimization problem with complex objective function and constraints. Such problems are NP-hard in general. Since our problem does not appear to posses any special simplifying structure, it is likely also NP-hard (although a formal proof is not given in this paper). For such complex optimization problem, metaheuristic algorithms (e.g., Genetic Algorithms [2], [11]) may be applied. But it is well known that such an approach cannot offer any performance guarantee on the final solution. That is, a heuristic or metaheuristic algorithm cannot offer any theoretical guarantee on the gap between its solution and the true optimal.

In this paper, we present a solution that offers theoretical guarantees on its optimality. The main contribution of this paper is a branch-and-bound-based solution procedure, embedded with a novel Reformulation-Linearization Technique 
(RLT) [18], that can produce $(1-\epsilon)$-optimal solution to the joint path selection and rate allocation problem. This result is significant as it not only provides theoretical understanding on this problem, but also offers a performance benchmark for any future proposed distributed algorithm and protocol for this problem.

The remainder of this paper is organized as follows. In Section II, we present problem formulation. In Section III, we present a branch-and-bound and RLT-based solution procedure. Implementation consideration is discussed in Section IV. In Section V, we present simulation results to demonstrate the efficacy of the solution procedure. We discuss related work in Section VI and Section VII concludes this paper.

\section{PROBLEM Formulation}

We consider a wireless mesh network consisting of a set of $\mathcal{N}$ nodes. We assume connectivity exists between a pair of nodes if each node falls within the transmission range of the other node. We further assume that some scheduling mechanism is in place or some physical layer mechanisms are employed such that the nodes are not interfering with each other during transmission. One example of this is in a multi-channel multi-radio (MC-MR) environment, where once the channels are properly assigned among the radios, the transmission among the nodes do not interfere with its neighboring nodes. Another example is that each node uses OFDM at physical/MAC layer. By properly assigning the frequency carrier at each node, we can eliminate interference among the nodes. In this context, we can model such wireless mesh network as a graph $\mathcal{G}(\mathcal{N}, \mathcal{L})$, where $\mathcal{L}$ is the set of wireless links. Further, the capacity on each wireless link can also be computed. We also assume the mean packet loss probability on link $\{i, j\}$ (e.g., due to transmission errors) is $p_{i j}$.

Consider a set of video communication sessions $\mathcal{S}$ in this network. Each video session $\sigma \in \mathcal{S}$ has a source node $z_{\sigma}$ and a destination node $d_{\sigma}$. For each session (i.e., a source-destination pair) $z_{\sigma}-d_{\sigma}$, there is a set of given paths, denoted by $\mathcal{P}_{\sigma}$. The total rate of a video stream, $R_{\sigma}$, originating at source node $z_{\sigma}$, is bounded by $\underline{R}_{\sigma} \leq R_{\sigma} \leq \bar{R}_{\sigma}, \sigma \in \mathcal{S}$, while the lower and upper bounds on $R_{\sigma}$ are determined by the specific video encoder and the video sequence used by source node $z_{\sigma}$. This rate $R_{\sigma}$ is to be split among the paths in $\mathcal{P}_{\sigma}$. Note that if our final solution assigns a rate of zero to a particular path in $\mathcal{P}_{\sigma}$, then we can interpret that this path is not selected. In this sense, rate allocation also correlates with path selection. Denoting an element in the rate vector be $R_{\sigma}^{h}, h \in \mathcal{P}_{\sigma}$, the following conditions must be satisfied:

$$
\sum_{h \in \mathcal{P}_{\sigma}} R_{\sigma}^{h}=R_{\sigma}, \text { and } R_{\sigma}^{h} \geq 0, \quad \forall h \in \mathcal{P}_{\sigma}, \quad \forall \sigma \in \mathcal{S} .
$$

Table I summarizes notation used in this paper.

\section{A. System Modeling}

We derive link and path statistics in this section. These statistics will be used to compute the application layer video distortion in Section II-B.

1) Load on a Link: The traffic on a link $\{i, j\}$ is the aggregate of traffic from different paths that traverse the link. To account for the potential packet loss at upstream links, let $\overline{\mathcal{P}}_{\sigma}^{h, i j}$ denote
TABLE I

NOTATION

\begin{tabular}{|l|l|}
\hline Symbol & Definition \\
\hline $\mathcal{N}$ & Set of nodes in the network \\
$\mathcal{L}$ & Set of links in the network \\
$\{i, j\}$ & Link from node $i$ to node $j$ \\
$c_{i j}$ & Capacity of link $\{i, j\}$ \\
$p_{i j}$ & Packet loss probability on link $\{i, j\}$ \\
$\lambda_{i j}$ & Average aggregate traffic rate on link $\{i, j\}$ \\
$\rho_{i j}$ & Utilization of link $\{i, j\}$ \\
$t_{i j}$ & Delay on link $\{i, j\}$ \\
$f_{i j}(y)$ & Probability density function of $t_{i j}$ \\
$M_{i j}(s)$ & Moment generating function of $t_{i j}$ \\
$M_{\sigma}^{h}(s)$ & Moment generating function of $T_{\sigma}^{h}$ \\
$\mathcal{S}$ & Set of video sessions in the network \\
$z_{\sigma}$ & Source node of session $\sigma$ \\
$d_{\sigma}$ & Destination node of session $\sigma$ \\
$\mathcal{P}_{\sigma}$ & Path set of session $\sigma$, from $z_{\sigma}$ to $d_{\sigma}$ \\
$\mathcal{P}_{\sigma}^{h}$ & A path in the set $\mathcal{P}_{\sigma}$, from $z_{\sigma}$ to $d_{\sigma}$ \\
$\Delta_{\sigma}$ & Decoding deadline of session $\sigma$ \\
$T_{\sigma}^{h}$ & End-to-end delay on path $\mathcal{P}_{\sigma}^{h} \in \mathcal{P}_{\sigma}$ \\
$T_{\sigma}$ & Weighted end-to-end delay for session $\sigma$ \\
$p_{\sigma}^{h}$ & End-to-end loss rate of $\mathcal{P}_{\sigma}^{h} \in \mathcal{P}_{\sigma}$ \\
$R_{\sigma}^{h}$ & Rate of video session $\sigma$ on path $\mathcal{P}_{\sigma}^{h} \in \mathcal{P} \sigma$ \\
$R_{\sigma}$ & Rate of video session $\sigma$ \\
$\bar{R}_{\sigma}$ & Maximum rate of video session $\sigma$ \\
$R_{\sigma}$ & Dinimum rate of video session $\sigma$ \\
$D_{\sigma}^{e}$ & Distortion caused by packet losses of session $\sigma$ \\
$D_{\sigma}^{e n c}$ & \\
$D_{\sigma}^{c g}$ & $D_{\sigma}^{l o s s}$
\end{tabular}

the upstream partial path for path $\mathcal{P}_{\sigma}^{h}$ up to (exclusive) link $\{i, j\}$. If link $\{i, j\} \notin \mathcal{P}_{\sigma}^{h}$, we have $\overline{\mathcal{P}}_{\sigma}^{h, i j}=\emptyset$. Then, the average rate of the aggregate traffic on link $\{i, j\} \in \mathcal{L}$ is

$$
\lambda_{i j}=\sum_{\sigma \in \mathcal{S}} \sum_{h \in \mathcal{P}_{\sigma}}\left[\prod_{\{l, m\} \in \overline{\mathcal{P}}_{\sigma}^{h, i j}}\left(1-p_{l m}\right)\right] \cdot R_{\sigma}^{h} .
$$

In other words, the traffic rate on link $\{i, j\}$ is the sum of rates of the video sessions that pass through this link, minus the loss incurred in their upstream links before reaching link $\{i, j\}$. The utilization of link $\{i, j\}$ is $\rho_{i j}=\lambda_{i j} / c_{i j}$.

2) Delay on a Link: To consider link delays due to congestion, we model each link $\{i, j\}$ as a general queueing system, with the input rate $\lambda_{i j}$ (defined in Section II-A1) and service capacity $c_{i j}$. Let $f_{i j}(y)$ be the probability density function for the queueing delay $t_{i j}$ on link $\{i, j\}$. We assume that all the moments of $t_{i j}$ are finite, which is true for most queueing systems.

For constant bit rate (CBR) video traffic that exhibits shortrange dependent (SRD) characteristics, ${ }^{1}$ we could model the queueing delay via an exponential distribution, i.e.,

$$
f_{i j}(y)=\alpha_{i j} \cdot e^{-\alpha_{i j} y}, \quad \text { for } y \geq 0,
$$

where $\alpha_{i j}=\left(c_{i j}-\lambda_{i j}\right)$ is the residual bandwidth on the link. For variable bit rate (VBR) video that exhibits long-range dependent (LRD) characteristics, we could model the link as a fractional Brownian motion (fBm) queue, where $t_{i j}$ has a heavy-

${ }^{1}$ Note that CBR does not necessarily imply a constant instantaneous rate. For CBR video, the bit rate is constant at large time scales, but at medium or findgrain time scales, an on-off pattern or back-to-back packet trains are often seen It is observed in a recent measurement study [10] that most CBR-coded videos are not long-range dependent (LRD). 
tailed Weibull distribution [14]. In this paper, we will focus on CBR video since it is used in most video communication applications.

3) Path Delay: Recall that for each session $\sigma \in \mathcal{S}$, there is a set of paths $\mathcal{P}_{\sigma}$ between the source node $z_{\sigma}$ and the destination node $d_{\sigma}$. Denote $T_{\sigma}^{h}$ the delay on path $\mathcal{P}_{\sigma}^{h}$ and $T_{\sigma}$ the weighted end-to-end delay of all the paths for session $\sigma$, respectively. We have

$$
T_{\sigma}=\sum_{h \in \mathcal{P}_{\sigma}} \frac{R_{\sigma}^{h}}{R_{\sigma}} \cdot T_{\sigma}^{h}
$$

Since the delay on path $\mathcal{P}_{\sigma}^{h}$ consists of delays on all the links along the path, we have that $T_{\sigma}^{h}=\sum_{\{i, j\} \in \mathcal{P}_{\sigma}^{h}} t_{i j}$.

For end-to-end delay, we apply the Chernoff Bound [5] to obtain a good approximation [7]. In the following, we illustrate such an approximation when link delays are exponentially distributed.

First, the moment generating function of $t_{i j}$ can be derived as follows:

$$
M_{i j}(s)=\mathrm{E}\left[e^{s \cdot t_{i j}}\right]=\frac{\alpha_{i j}}{\alpha_{i j}-s}, \quad \text { for } s<\alpha_{i j} .
$$

The moment generating function of $T_{\sigma}^{h}$ is

$$
M_{\sigma}^{h}(s)=\prod_{\{i, j\} \in \mathcal{P}_{\sigma}^{h}} M_{i j}(s), \quad \text { for } s<\min _{\{i, j\} \in \mathcal{P}_{\sigma}^{h}}\left\{\alpha_{i j}\right\} .
$$

Denote the decoding deadline as $\Delta_{\sigma}$ for session $\sigma$. We define $F_{\sigma}^{h}(s)$ as

$$
\begin{aligned}
F_{\sigma}^{h}(s)=s \cdot \Delta_{\sigma}- & \sum_{\{i, j\} \in \mathcal{P}_{\sigma}^{h}} \log M_{i j}(s), \\
& \text { for } s<\min _{\{i, j\} \in \mathcal{P}_{\sigma}^{h}}\left\{\alpha_{i j}\right\} .
\end{aligned}
$$

Since $F_{\sigma}^{h^{\prime \prime}}(s)<0$ for $s<\min _{\{i, j\} \in \mathcal{P}_{\sigma}^{h}}\left\{\alpha_{i j}\right\}, F_{\sigma}^{h}(s)$ is a strictly concave function with a unique maximum at $s_{\sigma, h}^{*}$. To avoid the trivial case of an intolerable video quality, we assume that $\Delta_{\sigma}>\mathrm{E}\left(T_{\sigma}^{h}\right)$ for any usable path. We can thus determine $s_{\sigma, h}^{*}$ by solving

$$
F_{\sigma}^{h^{\prime}}(s)=\Delta_{\sigma}-\sum_{\{i, j\} \in \mathcal{P}_{\sigma}^{h}} \frac{1}{\alpha_{i j}-s}=0 .
$$

Since $F_{\sigma}^{h^{\prime}}\left(\min _{\{i, j\} \in \mathcal{P}_{\sigma}^{h}}\left\{\alpha_{i j}\right\}\right)=-\infty<0$ and $F_{\sigma}^{h^{\prime}}(0)=\Delta_{\sigma}-$ $\mathrm{E}\left(T_{\sigma}^{h}\right)>0$, we have that $0<s_{\sigma, h}^{*}<\min _{\{i, j\} \in \mathcal{P}_{\sigma}^{h}}\left\{\alpha_{i j}\right\}$.

Applying the Chernoff Bound, the distribution of $T_{\sigma}^{h}$ can be approximated as [7]

$$
\operatorname{Pr}\left\{T_{\sigma}^{h} \geq \Delta_{\sigma}\right\} \approx\left(\frac{\exp \left\{-F_{\sigma}^{h}\left(s_{\sigma, h}^{*}\right)\right\}}{s_{\sigma, h}^{*} \delta_{\sigma, h}\left(s_{\sigma, h}^{*}\right) \sqrt{2 \pi}}\right)
$$

where $\delta_{\sigma, h}(s)=\sqrt{\partial^{2} \log M_{\sigma}^{h} / \partial s^{2}}$.

4) End-to-End Loss Rate: Assuming that the packet loss processes on the links are independent, the end-to-end loss probability for the path $\mathcal{P}_{\sigma}^{h} \in \mathcal{P}_{\sigma}$ can be approximated as

$$
p_{\sigma}^{h}=1-\prod_{\{i, j\} \in \mathcal{P}_{\sigma}^{h}}\left(1-p_{i j}\right), \quad \forall h \in \mathcal{P}_{\sigma}, \quad \forall \sigma \in \mathcal{S} .
$$

\section{B. Video Performance Characterization}

In [19], Stuhlmuller et al. developed an empirical rate-distortion model for a hybrid motion compensated video encoder. For a video sequence encoded at a target coding rate $R_{\sigma}$, the average end-to-end distortion $D_{\sigma}^{e}$ consists of the distortion at the encoder $\left(D_{\sigma}^{\mathrm{enc}}\right)$, the distortion due to congestion $\left(D_{\sigma}^{\mathrm{cg}}\right)$, and the distortion due to packet loss $\left(D_{\sigma}^{\text {loss }}\right)$. That is, $D_{\sigma}^{e}=$ $D_{\sigma}^{\text {enc }}+D_{\sigma}^{\text {cg }}+D_{\sigma}^{\text {loss }}$. Using the results for link and path statistics in Section II-A, we have

$$
\begin{array}{r}
\underbrace{e}_{\sigma}=\underbrace{D_{0}+\frac{\omega}{R_{\sigma}-R_{0}}}_{D_{\sigma}^{\text {enc }}}+\underbrace{\kappa \sum_{h \in \mathcal{P}_{\sigma}} \frac{R_{\sigma}^{h}}{R_{\sigma}}\left(1-p_{\sigma}^{h}\right) \operatorname{Pr}\left(T_{\sigma}^{h}>\Delta_{\sigma}\right)}_{D_{\sigma}^{\text {cg }}} \\
+\underbrace{\kappa \sum_{h \in \mathcal{P}_{\sigma}} \frac{R_{\sigma}^{h}}{R_{\sigma}} p_{\sigma}^{h}}_{D_{\sigma}^{\text {loss }}}
\end{array}
$$

where $D_{0}, \omega, R_{0}$, and $\kappa$ are constants for a specific video codec and video sequence. Since this model takes into account the effects of intra coding and spatial loop filtering, it provides accurate estimates for end-to-end distortion [19].

\section{Problem Formulation}

We are now ready to formulate the problem of optimal path selection and rate allocation for multiple video sessions (OPTPSRA). Our objective is to minimize application layer video distortion. Mathematically, problem OPT-PSRA can be stated in (4)-(10), shown at the bottom of the next page.

The objective function (4) is the sum of the average distortion of all the video sessions in the network. Alternative objective functions, such as minimizing the maximum of $\left\{D_{\sigma}^{e}\right\}$ among all sessions, or minimizing a function in the form of $\sum_{\sigma} f\left(D_{\sigma}^{e}\right)$ (e.g., a logarithmic utility function), can also be used. More details on this will be discussed in Section III-F.

The search space for problem OPT-PSRA consists of two sets of continuous variables: $i)$ the set of rates for all video sessions $\left\{R_{\sigma}\right\}_{\sigma \in \mathcal{S}}$; and ii) for a given session $\sigma \in \mathcal{S}$, the rate distribution among its paths $\mathcal{P}_{\sigma}$. Equation (5) provides the relationship between these two sets of variables. Equations (6) and (7) define the feasible region for the optimization variables. Equation (8) is the stability condition, which ensures that the links are stable with finite delays. Equation (9) is derived from the definition of $\delta_{\sigma, h}$ given in Section II-A, while (10) is a reformulation of (1) and is used to compute $s_{\sigma, h}^{*}$ for each path.

Observe that the objective function (4) and the constraints (9) and (10) are non-convex functions of $\left\{R_{\sigma}\right\}_{\sigma \in \mathcal{S}}$ and $\left\{R_{\sigma}^{h}\right\}_{h \in \mathcal{P}_{\sigma}}$. The rates of all the video sessions are closely coupled in (4). Since such problems are NP-hard in general [9], and problem OPT-PSRA does not appear to possess any special simplifying structure, it is likely to be NP-hard, although a formal proof is not given in this paper. In the following section, we present a solution procedure based on branch-and-bound framework for problem OPT-PSRA. Our proposed solution procedure can produce a solution within a normalized error of $\epsilon$ to the global optimum, where $\epsilon$ can be made arbitrarily small depending on the desired accuracy. 


\section{SOLUTION PROCEDURE}

Our solution procedure is based on a branch-and-bound framework, which embeds a novel relaxation technique called the Reformulation-Linearization Technique (RLT). In this section, we first reformulate the non-polynomial terms in problem OPT-PSRA so as to convert the formulation into a polynomial, nonlinear optimization problem. Then, we present details of the RLT-relaxation of this polynomial problem and the branch-and-bound solution procedure.

\section{A. Reformulation}

Due to the existence of non-polynomial terms in problem OPT-PSRA, our first goal is to reformulate this problem as a polynomial programming problem, which will greatly simplify the objective function as well as the constraints.

In the objective function (4), there are three sets of non-polynomial terms. In order to transform the first two non-polynomial terms, we define new variables $u_{\sigma}=1 /\left(R_{\sigma}-R_{0}\right)$ and $w_{\sigma}=\left(1 / R_{\sigma}\right) \cdot \sum_{h \in \mathcal{P}_{\sigma}} R_{\sigma}^{h} \cdot p_{\sigma}^{h}$. Substituting $u_{\sigma}$ and $w_{\sigma}$ into the objective function, we get two linear terms $\omega \cdot u_{\sigma}$ and $\kappa$. $w_{\sigma}$, respectively, and two sets of new polynomial constraints $u_{\sigma} \cdot\left(R_{\sigma}-R_{0}\right)=1$ and $w_{\sigma} \cdot R_{\sigma}=\sum_{h \in \mathcal{P}_{\sigma}} R_{\sigma}^{h} \cdot p_{\sigma}^{h}$.
In order to transform the third non-polynomial term in (4) which is a product of fractions, recall that $\alpha_{i j}$ denotes the residual bandwidth on link $\{i, j\}$, i.e.,

$$
\alpha_{i j}=c_{i j}-\sum_{\sigma \in \mathcal{S}} \sum_{h \in \mathcal{P}_{\sigma}} \theta_{i j}^{\sigma, h} \cdot R_{\sigma}^{h}
$$

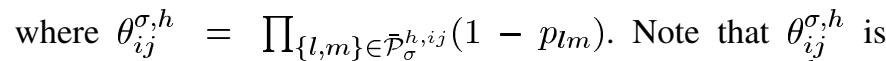
a constant, since all the paths are given. Denote $g_{\sigma}^{h}$ the weighted packet overdue probability due to path $\mathcal{P}_{\sigma}^{h}$, i.e., $g_{\sigma}^{h}=\left(R_{\sigma}^{h} / R_{\sigma}\right) \cdot \mathrm{P}\left(T_{\sigma}^{h}>\Delta_{\sigma}\right)$. Again, define a substitution variable $v_{i j}=1 /\left(\alpha_{i j}-s_{\sigma, h}^{*}\right)$ to convert the fractions in $\mathrm{P}\left(T_{\sigma}^{h}>\Delta_{\sigma}\right)$ to polynomial form, i.e.,

$$
g_{\sigma}^{h}=\frac{R_{\sigma}^{h}}{R_{\sigma}} \cdot\left[\frac{e^{-s_{\sigma, h}^{*} \Delta_{\sigma}}}{s_{\sigma, h}^{*} \delta_{\sigma, h} \sqrt{2 \pi}} \prod_{\{i, j\} \in \mathcal{P}_{\sigma}^{h}} \alpha_{i j} \cdot v_{i j}\right]
$$

This product form motivates us to apply logarithms on both sides of (12), which will lead to a linear constraint. This way, the complexity in the objective function can be effectively shifted into the constraints.

\section{OPT-PSRA}

$$
\begin{aligned}
& \text { Minimize } \\
& D=\sum_{\sigma \in \mathcal{S}}\left\{D_{0}+\frac{\omega}{R_{\sigma}-R_{0}}+\kappa \sum_{h \in \mathcal{P}_{\sigma}} \frac{R_{\sigma}^{h}}{R_{\sigma}}\left\{p_{\sigma}^{h}+\left(1-p_{\sigma}^{h}\right)\left\{\frac{e^{-s_{\sigma, h}^{*} \Delta_{\sigma}}}{s_{\sigma, h}^{*} \delta_{\sigma, h}\left(s_{\sigma, h}^{*}\right) \sqrt{2 \pi}}\right.\right.\right. \\
& \left.\left.\left.\prod_{\{i, j\} \in \mathcal{P}_{\sigma}^{h}} \frac{c_{i j}-\sum_{\varphi \in \mathcal{S}} \sum_{k \in \mathcal{P}_{\varphi}}\left[\prod_{\{l, m\} \in \overline{\mathcal{P}}_{\varphi}^{k, i j}}\left(1-p_{l m}\right)\right] R_{\varphi}^{k}}{c_{i j}-\sum_{\varphi \in \mathcal{S}} \sum_{k \in \mathcal{P}_{\varphi}}\left[\prod_{\{l, m\} \in \overline{\mathcal{P}}_{\varphi}^{k, i j}}\left(1-p_{l m}\right)\right] R_{\varphi}^{k}-s_{\sigma, h}^{*}}\right\}\right\}\right\} \\
& \text { subject to } \\
& R_{\sigma}=\sum_{h \in \mathcal{P}_{\sigma}} R_{\sigma}^{h}, \quad \text { for } \sigma \in \mathcal{S} \\
& \underline{R}_{\sigma} \leq R_{\sigma} \leq \bar{R}_{\sigma}, \quad \text { for } \sigma \in \mathcal{S} \\
& 0 \leq R_{\sigma}^{h} \leq R_{\sigma}, \quad \text { for } h \in \mathcal{P}_{\sigma}, \sigma \in \mathcal{S} \\
& \sum_{\sigma \in \mathcal{S}} \sum_{h \in \mathcal{P}_{\sigma}}\left[\prod_{\{l, m\} \in \overline{\mathcal{P}}_{\sigma}^{h, i j}}\left(1-p_{l m}\right)\right] \cdot R_{\sigma}^{h} \leq(1-\tau) \cdot c_{i j}, \quad \text { for }\{i, j\} \in \mathcal{P}_{\sigma} \\
& \delta_{\sigma, h}^{2}=\sum_{\{i, j\} \in \mathcal{P}_{\sigma}^{h}} \frac{1}{\left(c_{i j}-\sum_{\varphi \in \mathcal{S}} \sum_{k \in \mathcal{P}_{\varphi}}\left[\prod_{\{l, m\} \in \overline{\mathcal{P}}_{\varphi}^{k, i j}}\left(1-p_{l m}\right)\right] R_{\varphi}^{k}-s_{\sigma, h}^{*}\right)^{2}}, \quad \text { for } h \in \mathcal{P}_{\sigma}, \forall \sigma \in \mathcal{S} \\
& \Delta_{\sigma}=\sum_{\{i, j\} \in \mathcal{P}_{\sigma}^{h}} \frac{1}{c_{i j}-\sum_{\varphi \in \mathcal{S}} \sum_{k \in \mathcal{P}_{\varphi}}\left[\prod_{\{l, m\} \in \overline{\mathcal{P}}_{\varphi}^{k, i j}}\left(1-p_{l m}\right)\right] R_{\varphi}^{k}-s_{\sigma, h}^{*}}, \quad \text { for } h \in \mathcal{P}_{\sigma}, \forall \sigma \in \mathcal{S} \text {. }
\end{aligned}
$$




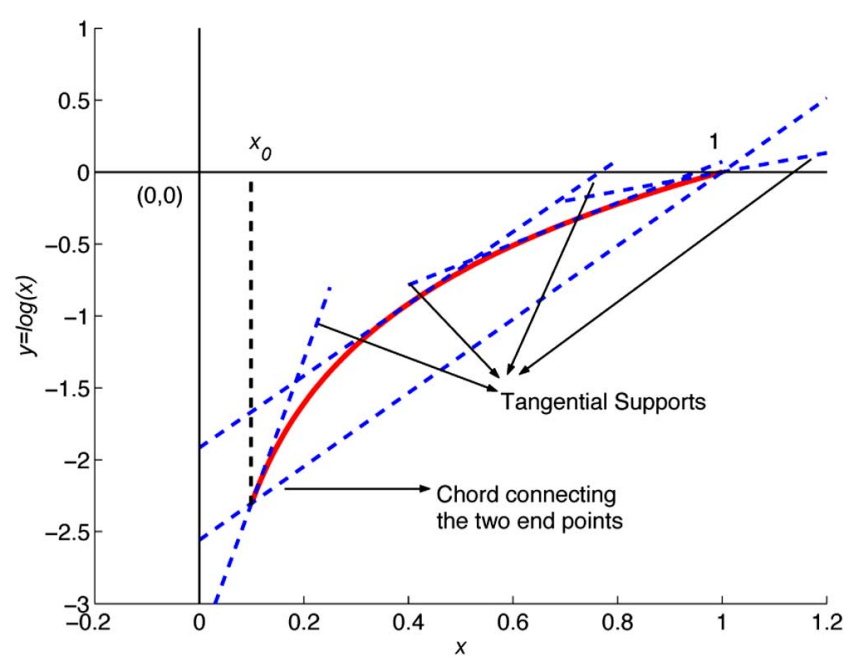

Fig. 1. Polyhedral outer approximation for $y=\log (x)$ in $0<x_{0} \leq x \leq 1$.

Taking logarithms on both sides of (12) and making the following substitutions:

$$
\begin{cases}\gamma_{\sigma, h}^{g}=\log \left(g_{\sigma}^{h}\right), & \text { for } \sigma \in \mathcal{S} \\ \gamma_{\sigma, h}^{R}=\log \left(R_{\sigma}^{h}\right), & \text { for } \sigma \in \mathcal{S} \\ \gamma_{\sigma}^{R}=\log \left(R_{\sigma}\right), & \text { for } \sigma \in \mathcal{S} \\ \gamma_{\sigma, h}^{s}=\log \left(s_{\sigma, h}^{*}\right), & \text { for } \sigma \in \mathcal{S} \\ \gamma_{\sigma, h}^{\delta}=\log \left(\delta_{\sigma, h}\right), & \text { for } \sigma \in \mathcal{S} \\ \gamma_{i j}^{\alpha}=\log \left(\alpha_{i j}\right), & \text { for }\{i, j\} \in \mathcal{P}_{\sigma}, \sigma \in \mathcal{S} \\ \gamma_{i j}^{v}=\log \left(v_{i j}\right), & \text { for }\{i, j\} \in \mathcal{P}_{\sigma}, \sigma \in \mathcal{S}\end{cases}
$$

we have $\gamma_{\sigma, h}^{g}=\gamma_{\sigma, h}^{R}-\gamma_{\sigma}^{R}-s_{\sigma, h}^{*} \Delta_{\sigma}-\gamma_{\sigma, h}^{s}-\gamma_{\sigma, h}^{\delta}+$ $\sum_{\{i, j\} \in \mathcal{P}_{\sigma}^{h}}\left(\gamma_{i j}^{\alpha}+\gamma_{i j}^{v}\right)-\log (\sqrt{2 \pi})$, where $\log (\cdot)$ denotes logarithm to the natural base $e$.

Once the objective function is simplified, we now deal with the new constraints of the form $y=\log (x)$, as shown in (13). We can linearize this logarithmic relationship over some suitable tightly bounded interval using a polyhedral outer approximation that is comprised of a convex envelope in concert with several tangential supports. For instance, if $x$ is bounded by $0<x_{0} \leq x \leq 1$, these constraints can be written as follows:

$$
\left\{\begin{array}{l}
y \geq \frac{\log \left(x_{0}\right)}{1-x_{0}} \cdot(1-x) \\
y \leq \log \left(x_{k}\right)+\frac{x-x_{k}}{x_{k}}, \quad k=0, \ldots, k_{\max }-1
\end{array}\right.
$$

where $x_{k}=x_{0}+\left(1-x_{0}\right) \cdot k /\left(k_{\max }-1\right)$, for $k=$ $0,1,2, \ldots, k_{\max }-1$. A four-point tangential approximation can be obtained by letting $k_{\max }=4$, as illustrated in Fig. 1 . The corresponding convex envelope consists of a chord connecting the two end points, which is used in combination with tangential supports at four points including the two end points. As a result, every logarithmic relationship specified in (13) translates to five linear constraints constituting a polyhedral outer approximation. Note that such polyhedral outer approximations will be iteratively tightened during the branch-and-bound procedure (see Section III-B).

Now we have successfully reformulated the objective function into a linear form, and introduced the corresponding polynomial constraints to make the reformulation tight. To make the problem polynomial, it remains to transform the two constraints (9) and (10) to a polynomial form. From the definition of $v_{i j}$, we have that $\delta_{\sigma, h}^{2}=\sum_{\{i, j\} \in \mathcal{P}_{\sigma}^{h}} v_{i j}^{2}$ and $\Delta_{\sigma}=\sum_{\{i, j\} \in \mathcal{P}^{h}} v_{i j}$. With the above reformulation, we can now rewrite problem OPT-PSRA as the following polynomial programming problem ( $p$-PSRA).

$$
\begin{aligned}
& \frac{p \text { - PSRA }}{\text { Minimize }} \\
& D\left(x_{k}\right)=\sum_{\sigma \in \mathcal{S}}\left\{D_{0}+\omega u_{\sigma}+\kappa w_{\sigma}+\kappa \sum_{n \in \mathcal{P}_{\sigma}}\left(1-p_{\sigma}^{h}\right) g_{\sigma}^{h}\right\} \\
& \text { subject to } \\
& R_{\sigma}=\sum_{h \in \mathcal{P}_{\sigma}} R_{\sigma}^{h}, \quad \text { for } \sigma \in \mathcal{S} \\
& 0 \leq R_{\sigma}^{h} \leq R_{\sigma}, \quad \text { for } h \in \mathcal{P}_{\sigma}, \sigma \in \mathcal{S} \\
& \alpha_{i j}=c_{i j}-\sum_{\varphi \in \mathcal{S}} \sum_{k \in \mathcal{P}_{\varphi}} \theta_{i j}^{\varphi, k} \cdot R_{\varphi}^{k}, \quad \text { for }\{i, j\} \in \mathcal{P}_{\sigma} \\
& \alpha_{i j} \geq \tau \cdot c_{i j}, \quad \text { for }\{i, j\} \in \mathcal{P}_{\sigma} \\
& \Delta_{\sigma}=\sum_{\{i, j\} \in \mathcal{P}_{\sigma}^{h}} v_{i j}, \quad \text { for } h \in \mathcal{P}_{\sigma}, \sigma \in \mathcal{S} \\
& v_{i j} \cdot\left(\alpha_{i j}-s_{\sigma, h}^{*}\right)=1, \quad \text { for }\{i, j\} \in \mathcal{P}_{\sigma}, \sigma \in \mathcal{S} \\
& \delta_{\sigma, h}^{2}=\sum_{\{i, j\} \in \mathcal{P}_{\sigma}^{h}} v_{i j}^{2}, \quad \text { for } h \in \mathcal{P}_{\sigma}, \sigma \in \mathcal{S} \\
& w_{\sigma} \cdot R_{\sigma}=\sum_{h \in \mathcal{P}_{\sigma}} R_{\sigma}^{h} \cdot p_{\sigma}^{h}, \quad \text { for } \sigma \in \mathcal{S} \\
& u_{\sigma} \cdot\left(R_{\sigma}-R_{0}\right)=1, \quad \text { for } \sigma \in \mathcal{S} \\
& \gamma_{\sigma, h}^{g}=\gamma_{\sigma, h}^{R}-\gamma_{\sigma}^{R}-s_{\sigma, h}^{*} \Delta_{\sigma}-\gamma_{\sigma, h}^{s}-\gamma_{\sigma, h}^{\delta} \\
& \quad+\sum_{\{i, j\} \in \mathcal{P}_{\sigma}^{h}}\left(\gamma_{i j}^{\alpha}+\gamma_{i j}^{v}\right)-\log (\sqrt{2 \pi}) \\
& \quad \text { for } h \in \mathcal{P}_{\sigma}, \sigma \in \mathcal{S}
\end{aligned}
$$

Polyhedral outer approximations for

$$
\begin{aligned}
& \gamma_{\sigma, h}^{R}, \gamma_{\sigma}^{R}, \gamma_{\sigma, h}^{g}, \gamma_{\sigma, h}^{s}, \gamma_{\sigma, h}^{\delta}, \gamma_{i j}^{\alpha}, \gamma_{i j}^{v}, \\
& \quad \text { for }\{i, j\} \in \mathcal{P}_{\sigma}^{h}, h \in \mathcal{P}_{\sigma}, \sigma \in \mathcal{S}
\end{aligned}
$$

$\underline{R}_{\sigma} \leq R_{\sigma} \leq \bar{R}_{\sigma}, \quad$ for $\sigma \in \mathcal{S}$

$\frac{1}{\bar{R}_{\sigma}-R_{0}} \leq u_{\sigma} \leq \frac{1}{\underline{R}_{\sigma}-R_{0}}, \quad$ for $\sigma \in \mathcal{S}$

Implied bounds for all other variables.

In problem $p$-PSRA, as in the case of the original problem, constraints (15) and (16) are stability constraints and constraints (23) are bounds on video rates. Constraint (17) is a reformulation of constraint (10) of the original OPT-PSRA problem. Constraints (18)-(20) and (24) are derived from the definition of the corresponding variables. Constraint (21) results from a linearization of (12), and the constraints (22) are the polyhedral outer approximations for the logarithms of the packet overdue probabilities on the paths $\mathcal{P}_{\sigma}^{h} \in \mathcal{P}_{\sigma}$ [see (12)-(14)].

Problem OPT-PSRA is now transformed into a polynomial nonlinear programming problem (NLP) of order two. The highly complex objective function (4) is greatly simplified (i.e., linearized) and the complexity is shifted into the constraints in polynomial form. 
Although this problem is simpler than the original problem, it is still a quadratic polynomial programming problem, which is NP-hard in general [18]. In the remainder of this section, we present our branch-and-bound and RLT-based solution procedure to solve problem $p$-PSRA.

\section{B. Branch-and-Bound Framework}

Branch-and-bound is an iterative relaxation algorithm [18], which seeks to provide an $(1-\epsilon)$-optimal solution to a nonlinear programming problem by partitioning the original search space into smaller sub-hyperrectangles [18], and thereby solving the smaller sub-problems. Here $\epsilon$ denotes an arbitrarily small, prescribed constant reflecting our desired accuracy for the final optimal solution. In branch-and-bound, the original problem is first relaxed using a suitable relaxation technique to obtain an easier-to-solve, lower-bounding problem. In our approach, we choose the Reformulation-Linearization Technique (RLT) [17], [18] to obtain a linear programming (LP) relaxation (see Section III-C). The optimal solution to this LP relaxation provides a lower bound (LB) for the original problem. Since such an LB solution is likely to be infeasible to the original NLP problem, a local search algorithm (using the LB solution as a starting point) is employed to obtain a feasible solution to the original problem. This new feasible solution then provides an upper bound (UB) for the original problem.

During each iteration, the original problem $P$ is partitioned into sub-problems, each with a smaller search space. In particular, during each iteration, for a sub-problem in the branchand-bound tree, a branching process is carried out to obtain two new sub-problems. The partitioning of the original solution space, i.e., the branching rule, will be explained further in Section III-C. The sub-problems are inserted into a problem list $H$, which records the active sub-problems in the branch-andbound tree structure.

More specifically, in the beginning, the problem list $H$ is initialized with the original problem $P$. At any given iteration, the lower bound and upper bound for $P$ are computed as

$$
\left\{\begin{array}{l}
\mathrm{LB}=\min \left\{\mathrm{LB}_{k}: \text { Problem } k \in H\right\} \\
\mathrm{UB}=\min \left\{\mathrm{UB}_{k}: \text { Problem } k \in H\right\} .
\end{array}\right.
$$

The method proceeds by choosing the next problem to partition from the problem list. In our approach, the problem with the smallest LB is chosen. The corresponding problem $h$ is then partitioned into two sub-problems $h_{1}$ and $h_{2}$, which replace problem $h$ in problem list $H$. Every time a new sub-problem $h_{i}$ is added to the list, $\mathrm{LB}_{h_{i}}$ and $\mathrm{UB}_{h_{i}}$ are computed, and the LB and UB for the original problem $P$ are updated as in (25). At any given iteration, if $\mathrm{LB} \geq(1-\epsilon) \cdot \mathrm{UB}$, the procedure exits with an $(1-\epsilon)$-optimal solution. For any problem $h_{i}$ in the problem list $H$, if $\mathrm{LB}_{h_{i}} \geq(1-\epsilon) \cdot \mathrm{UB}$, we consider that this sub-problem can no longer produce an improving solution in the future branching process. Therefore, this node can be removed from the branch-and-bound tree. In this manner, the branch-and-bound can prune or fathom certain branches of the tree, without the need to solving all the subproblems in the tree to completion. Depending on the effectiveness of this pruning strategy, the branch-and-bound procedure can provide a solution much faster than a general divide-and-conquer approach.

\section{Relaxation With the Reformulation-Linearization Technique}

RLT is a relaxation technique that can be used to produce tight polyhedral outer approximations or linear programming relaxations for an underlying nonlinear, non-convex polynomial programming problem. This relaxation will provide a tight lower bound on a minimization problem [17], [18]. In our approach, the key to obtaining an $(1-\epsilon)$-optimal solution is to embed RLT into the branch-and-bound framework as discussed earlier, and to coordinate with it a suitable partitioning strategy that would enable the gap between the lower and upper bounds thus generated to iteratively converge.

In the RLT procedure, nonlinear implied constraints are generated by taking the products of bounding terms of the decision variables, up to a suitable order and also, possibly products of other defining constraints of the problem. The resulting problem is subsequently linearized by variable substitutions, one for each nonlinear term appearing in the problem, including both the objective function and the constraints. This automatically creates outer linearizations that approximate the closure of the convex hull of the feasible region $\Omega$.

For instance, the second order term $u_{\sigma} \cdot R_{\sigma}$ in (20) can be viewed as a single term, for which we can introduce a new variable $\mu_{\sigma}$, thereby substituting $\mu_{\sigma}=u_{\sigma} \cdot R_{\sigma}$. Since $u_{\sigma}$ and $R_{\sigma}$ are each bounded by $\left(u_{\sigma}\right)_{L} \leq u_{\sigma} \leq\left(u_{\sigma}\right)_{U}$ and $\left(R_{\sigma}\right)_{L} \leq R_{\sigma} \leq\left(R_{\sigma}\right)_{U}$, respectively, we generate the following relational constraints, which are known as RLT bound-factor product constraints.

$$
\left\{\begin{array}{l}
\left\{\left[u_{\sigma}-\left(u_{\sigma}\right)_{L}\right] \cdot\left[R_{\sigma}-\left(R_{\sigma}\right)_{L}\right]\right\}_{L S} \geq 0 \\
\left\{\left[u_{\sigma}-\left(u_{\sigma}\right)_{L}\right] \cdot\left[\left(R_{\sigma}\right)_{U}-R_{\sigma}\right]\right\}_{L S} \geq 0 \\
\left\{\left[\left(u_{\sigma}\right)_{U}-u_{\sigma}\right] \cdot\left[R_{\sigma}-\left(R_{\sigma}\right)_{L}\right]\right\}_{L S} \geq 0 \\
\left\{\left[\left(u_{\sigma}\right)_{U}-u_{\sigma}\right] \cdot\left[\left(R_{\sigma}\right)_{U}-R_{\sigma}\right]\right\}_{L S} \geq 0
\end{array}\right.
$$

where $\{\cdot\}_{L S}$ denotes a linearization step under the substitution $\mu_{\sigma}=u_{\sigma} \cdot R_{\sigma}$. From the above relationships and by substituting $\mu_{\sigma}=u_{\sigma} \cdot R_{\sigma}$, we obtain the following RLT constraints for $\mu_{\sigma}$ :

$$
\left\{\begin{array}{l}
\left(u_{\sigma}\right)_{L} \cdot R_{\sigma}+\left(R_{\sigma}\right)_{L} \cdot u_{\sigma}-\mu_{\sigma} \leq\left(u_{\sigma}\right)_{L} \cdot\left(R_{\sigma}\right)_{L} \\
\left(u_{\sigma}\right)_{U} \cdot R_{\sigma}+\left(R_{\sigma}\right)_{L} \cdot u_{\sigma}-\mu_{\sigma} \geq\left(u_{\sigma}\right)_{U} \cdot\left(R_{\sigma}\right)_{L} \\
\left(u_{\sigma}\right)_{L} \cdot R_{\sigma}+\left(R_{\sigma}\right)_{U} \cdot u_{\sigma}-\mu_{\sigma} \geq\left(u_{\sigma}\right)_{L} \cdot\left(R_{\sigma}\right)_{U} \\
\left(u_{\sigma}\right)_{U} \cdot R_{\sigma}+\left(R_{\sigma}\right)_{U} \cdot u_{\sigma}-\mu_{\sigma} \leq\left(u_{\sigma}\right)_{U} \cdot\left(R_{\sigma}\right)_{U} .
\end{array}\right.
$$

We therefore replace the second-order term $u_{\sigma} \cdot R_{\sigma}$ with the linear term $\mu_{\sigma}$ in (20) and introduce the above linear RLT bound-factor constraints for $\mu_{\sigma}$ into problem $p$-PSRA formulation. Similarly, we define new variables for all the remaining nonlinear terms in problem $p$-PSRA, including $\xi_{i j}=v_{i j} \cdot \alpha_{i j}$, $\psi_{i j}=v_{i j} \cdot s_{\sigma, h}^{*}, \phi_{i j}=v_{i j}^{2}, \chi_{\sigma, h}=\delta_{\sigma, h}^{2}$, and $\eta_{\sigma}=w_{\sigma} \cdot R_{\sigma}$, and make substitutions in the same manner.

It is worth noting that, when incorporating RLT into the branch-and-bound framework, the original bounding set $\Omega$ is decomposed into two corresponding hyper-rectangles, based on a so-called branching variable. In RLT, the discrepancy between an RLT variable and the corresponding nonlinear term that the RLT variable represents is called the relaxation error. In our solution procedure, the branching variable is chosen to be the variable that yields the largest relaxation error. Such a branching rule ensures that all the discrepancies will be driven to zero iteratively. 
After replacing all the nonlinear terms as above and adding the corresponding RLT constraints into problem $p$-PSRA formulation, we obtain the following linear programming ( $\ell$-PSRA), which can be efficiently solved in polynomial time.

\section{$\ell$ - PSRA}

\section{Minimize}

$$
D\left(x_{k}\right)=\sum_{\sigma \in \mathcal{S}}\left\{D_{0}+\omega u_{\sigma}+\kappa w_{\sigma}+\kappa \sum_{h \in \mathcal{P}_{\sigma}}\left(1-p_{\sigma}^{h}\right) g_{\sigma}^{h}\right\}
$$

\section{subject to}

$$
\begin{aligned}
& R_{\sigma}=\sum_{h \in \mathcal{P}_{\sigma}} R_{\sigma}^{h}, \quad \text { for } \sigma \in \mathcal{S} \\
& \alpha_{i j}=c_{i j}-\sum_{\varphi \in \mathcal{S}} \sum_{k \in \mathcal{P}_{\varphi}} \theta_{\varphi, i j} \cdot R_{\varphi}, \quad \text { for }\{i, j\} \in \mathcal{P}_{\sigma} \\
& \alpha_{i j} \geq \tau \cdot c_{i j}, \quad \text { for }\{i, j\} \in \mathcal{P}_{\sigma} \\
& \Delta_{\sigma}=\sum_{\{i, j\} \in \mathcal{P}_{\sigma}^{h}} v_{i j}, \quad \text { for } h \in \mathcal{P}_{\sigma}, \sigma \in \mathcal{S} \\
& \xi_{i j}-\psi_{i j}=1, \quad \text { for }\{i, j\} \in \mathcal{P}_{\sigma}, \sigma \in \mathcal{S} \\
& \chi_{\sigma, h}=\sum_{\{i, j\} \in \mathcal{P}_{\sigma}^{h}} \phi_{i j}, \quad \text { for } h \in \mathcal{P}_{\sigma}, \sigma \in \mathcal{S} \\
& \eta_{\sigma}=\sum_{h \in \mathcal{P}_{\sigma}} R_{\sigma}^{h} \cdot p_{\sigma}^{h}, \quad \text { for } \sigma \in \mathcal{S} \\
& \mu_{\sigma}-R_{0} \cdot u_{\sigma}=1, \quad \text { for } \sigma \in \mathcal{S} \\
& \gamma_{\sigma, h}^{g}=\gamma_{\sigma, h}^{R}-\gamma_{\sigma}^{R}-s_{\sigma, h}^{*} \Delta_{\sigma}-\gamma_{\sigma, h}^{s}-\gamma_{\sigma, h}^{\delta} \\
& \quad+\sum_{\{i, j\} \in \mathcal{P}_{\sigma}^{h}}\left(\gamma_{i j}^{\alpha}+\gamma_{i j}^{v}\right)-\log (\sqrt{2 \pi}), \\
& \quad \text { for } h \in \mathcal{P}_{\sigma}, \sigma \in \mathcal{S}
\end{aligned}
$$

Polyhedral outer approximations for

$$
\begin{aligned}
& \gamma_{\sigma, h}^{R}, \gamma_{\sigma}^{R}, \gamma_{\sigma, h}^{g}, \gamma_{\sigma, h}^{s}, \gamma_{\sigma, h}^{\delta}, \gamma_{i j}^{\alpha}, \gamma_{i j}^{v}, \\
& \quad \text { for }\{i, j\} \in \mathcal{P}_{\sigma}^{h}, h \in \mathcal{P}_{\sigma}, \sigma \in \mathcal{S}
\end{aligned}
$$

$\underline{R}_{\sigma} \leq R_{\sigma} \leq \bar{R}_{\sigma}, \quad$ for $\sigma \in \mathcal{S}$

$0 \leq R_{\sigma}^{h} \leq R_{\sigma}, \quad$ for $h \in \mathcal{P}_{\sigma}, \sigma \in \mathcal{S}$

$\frac{1}{\bar{R}_{\sigma}-R_{0}} \leq u_{\sigma} \leq \frac{1}{\underline{R}_{\sigma}-R_{0}}, \quad$ for $\sigma \in \mathcal{S}$

Bound-factor product RLT constraints for the terms

$$
\begin{aligned}
& \mu_{\sigma}=u_{\sigma} \cdot R_{\sigma}, \xi_{i j}=v_{i j} \cdot \alpha_{i j}, \psi_{i j}=v_{i j} \cdot s_{\sigma, h}^{*}, \\
& \phi_{i j}=v_{i j}^{2}, \chi_{\sigma, h}=\delta_{\sigma, h}^{2}, \text { and } \eta_{\sigma}=w_{\sigma} \cdot R_{\sigma}
\end{aligned}
$$

Implied bounds for all other variables.

\section{Partitioning Strategies}

The objective of the partitioning step is to find the branching variable that will enable us to split the search space $\Omega_{k}$ for problem $k$ into two search sub-spaces $\Omega_{k_{1}}$ and $\Omega_{k_{2}}$. For problem OPT-PSRA, we need to consider two classes of optimization variables for partitioning, i.e., the substitution variables (e.g., $\mu_{\sigma}$ ), and the logarithm substitution terms [e.g., $\gamma_{\sigma}^{R}$ in (13)].

In the case of branching decisions based on the substitution variables such as $\mu_{\sigma}=u_{\sigma} \cdot R_{\sigma}$, we first find the maximum relaxation error between the substitution variable and the corresponding product term, say, $\left|\hat{u}_{\sigma} \hat{R}_{\sigma}-\hat{\mu}_{\sigma}\right|$. We then verify whether the following condition is satisfied:

$$
\begin{aligned}
& \left.\left[\left(u_{\sigma}\right)_{U}-\left(u_{\sigma}\right)_{L}\right)\right] \cdot \min \left\{\hat{u}_{\sigma}-\left(u_{\sigma}\right)_{L},\left(u_{\sigma}\right)_{U}-\hat{u}_{\sigma}\right\} \\
& \left.\quad \geq\left[\left(R_{\sigma}\right)_{U}-\left(R_{\sigma}\right)_{L}\right)\right] \cdot \min \left\{\hat{R}_{\sigma}-\left(R_{\sigma}\right)_{L},\left(R_{\sigma}\right)_{U}-\hat{R}_{\sigma}\right\} .
\end{aligned}
$$

If this condition holds true, we partition the search space $\Omega_{k}$ of problem $k$ into two new sub-spaces $\Omega_{k_{1}}$ and $\Omega_{k_{2}}$, by dividing the range $\left[\left(u_{\sigma}\right)_{L},\left(u_{\sigma}\right)_{U}\right]$ into two subregions $\left[\left(u_{\sigma}\right)_{L}, \hat{u}_{\sigma}\right]$ and $\left[\hat{u}_{\sigma},\left(u_{\sigma}\right)_{U}\right]$. Otherwise, we partition $\Omega_{k}$ by dividing $\left[\left(R_{\sigma}\right)_{L},\left(R_{\sigma}\right)_{U}\right]$ into $\left[\left(R_{\sigma}\right)_{L}, \hat{R}_{\sigma}\right]$ and $\left[\hat{R}_{\sigma},\left(R_{\sigma}\right)_{U}\right]$.

This partition variable selection policy can be further improved by exploiting the physical interpretation of certain variables and weighing their significance. For example, based on the discussion in Section II-A3, we observe that $\alpha_{i j}$ 's are the most important set of variables that directly affect the computation of average distortion. Therefore, during the implementation of our RLT-based branching algorithm, we assign highest priority to these variables when selecting the partition variable. Note that the choice of partition variable does not alter the final result but it does play an important role in speeding up the convergence time of the algorithm.

Regarding $\gamma_{\sigma}^{R}$ in (13), we first find the variable that gives the greatest discrepancy between the logarithm value, say, $\log \left(\hat{R}_{\sigma}\right)$ and the left-hand side of the corresponding substitution [e.g., (13)] among all such terms, and then either bisect the interval of this variable (e.g., $\left.\left[\left(R_{\sigma}\right)_{L},\left(R_{\sigma}\right)_{U}\right]\right)$ evenly, or divide this interval at the point $\hat{R}_{\sigma}$.

\section{E. A Local Search Algorithm}

As discussed in Section III-B, in the branch-and-bound procedure, the solution to the relaxation problem is likely infeasible to the original problem. This problem can be resolved by finding a feasible solution to the original problem via a local search algorithm that starts from the infeasible solution.

For problem OPT-PSRA, we adopt a local search strategy that computes a feasible solution $(\mathbf{R}, \mathbf{u}, \mathbf{v}, \alpha, \delta, \mathbf{s})$ from the solution to the relaxation problem $(\hat{\mathbf{R}}, \hat{\mathbf{u}}, \hat{\mathbf{v}}, \hat{\alpha}, \hat{\delta}, \hat{\mathbf{s}})$. Specifically, since the rates of the video sessions obtained from the solution to the relaxation problem are always feasible to the original problem (i.e., the stability constraints are always satisfied and the rates are always within the lower and upper bounds), we let $\mathbf{R}=\hat{\mathbf{R}}$. From $\mathbf{R}$, we can compute the values of $\alpha_{i j}$ from (11), the values of $s_{\sigma, h}^{*}$ from (1), and the values of $u_{\sigma}$ from (20). After obtaining $\alpha_{i j}$ and $s_{\sigma, h}^{*}$, we can compute $v_{i j}$ from (18), and $\delta_{\sigma, h}$ from (19). Therefore, a feasible solution to the original problem OPT-PSRA, $(\mathbf{R}, \mathbf{u}, \mathbf{v}, \alpha, \delta, \mathbf{s})$, can be obtained from the solution to the relaxed problem.

Fig. 2 shows the complete solution procedure to our path selection and rate allocation problem.

\section{F. Extension to Other Objective Functions}

In problem OPT-PSRA, the objective is to minimize the sum of the average distortions of all the video sessions in the net- 


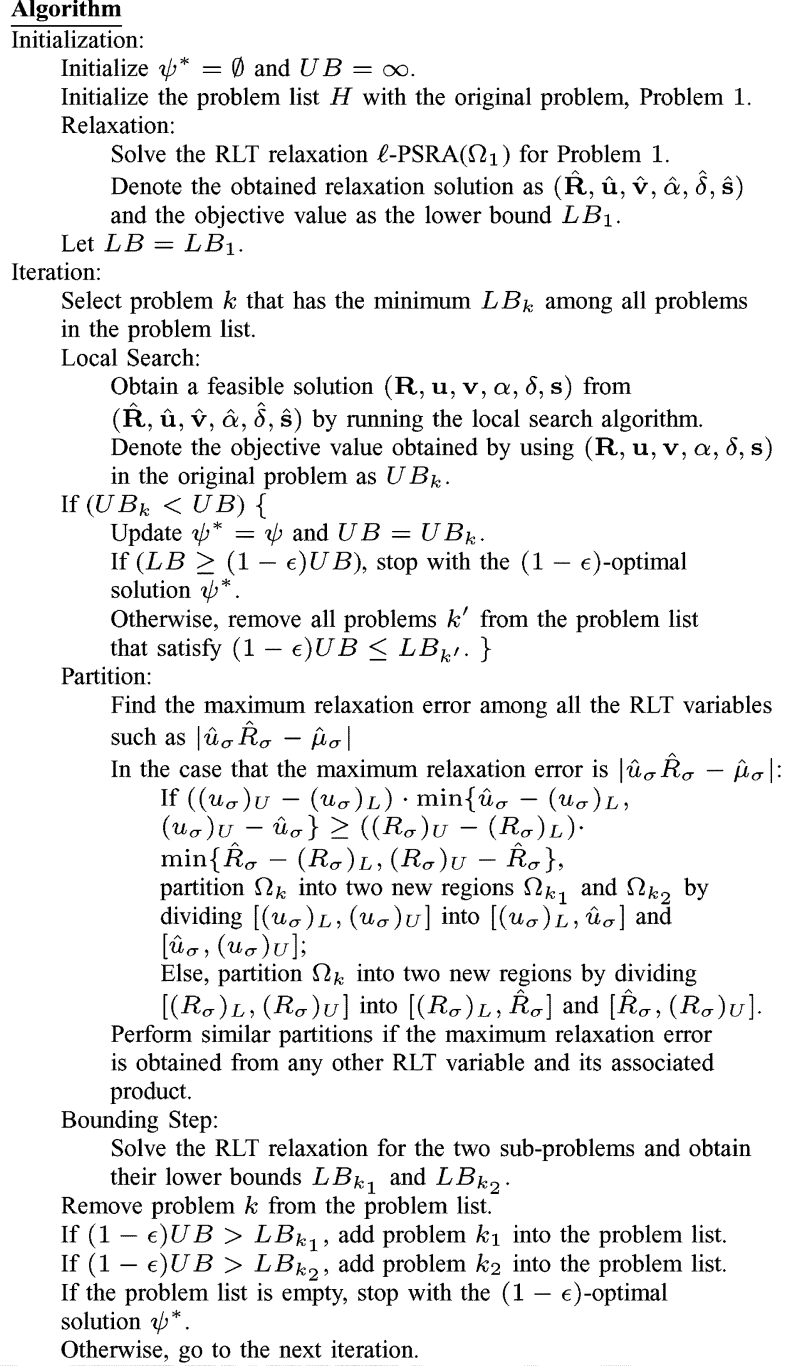

Fig. 2. Proposed solution procedure for problem OPT-PSRA.

work [see (4)]. We now show how the solution procedure can be extended to solve problems with other objective functions.

As discussed in Section II-C, we could use the following objective function in the problem formulation: minimize the maximum of $D_{\sigma}^{e}$ among all sessions $\sigma \in \mathcal{S}$. For this objective function, we can make a simple transformation by defining a new variable as $y=\max _{\sigma \in \mathcal{S}}\left\{D_{\sigma}^{e}\right\}$. The transformed problem minimizes $y$, with additional constraint $D_{\sigma}^{e} \leq y$ for all $\sigma \in \mathcal{S}$. Then, our branch-and-bound/RLT-based solution procedure can be applied to this transformed problem to obtain $(1-\epsilon)$-optimal solution.

As another example, the objective function could be to minimize $\sum_{\sigma \in \mathcal{S}} \log \left(D_{\sigma}^{e}\right)$. In this case, we can first make a transformation by defining new variables $y_{\sigma}=\log \left(D_{\sigma}^{e}\right)$, for all $\sigma \in \mathcal{S}$. The transformed problem minimizes $\sum_{\sigma \in \mathcal{S}} y_{\sigma}$, with additional constraints $y_{\sigma}=\log \left(D_{\sigma}^{e}\right)$, for all $\sigma \in \mathcal{S}$. Then, the polyhedral outer approximations could be applied to these logarithmic constraints (see Section III-A) and the branch-andbound/RLT-based solution procedure can be applied to solve the transformed problem.
As a final example, in some cases, it may be necessary to guarantee the performance of each video session (e.g., a distortion no larger than a predefined threshold), rather than achieving the best performance that the network can offer. For this objective function, we can add an additional constraint for each video session, in the form of $D_{\sigma}^{e} \leq y_{\sigma}$, where $y_{\sigma}$ is the predefined distortion threshold for session $\sigma \in \mathcal{S}$.

\section{IMPLEMENTATION CONSIDERATIONS}

The focus of this paper is on developing theoretical solution to a complex cross-layer optimization problem for video communications. In this regard, the solution proposed in this paper is centralized in nature. Nevertheless, we briefly discuss issues related to implementation.

For a small to moderate sized network involving long-lived video traffic, a centralized implementation based on the proposed solution may be feasible. Here, a centralized network server can be employed to maintain and update the network topology information, the global link state information and active video session information. This centralized network server is somewhat similar to the so-called Bandwidth Broker (BB) entity under the Internet DiffServ paradigm [6], [21]. In addition to computing $(1-\epsilon)$-optimal solution, this centralized network server can also be used to perform admission control and other important functions on the control plane. For the purpose of computing $(1-\epsilon)$-optimal solution, three types of information need to be maintained and updated, namely i) links statistics; ii) the available path set for each session; and iii) video specific parameters. Under this approach, link statistics can be measured by each node, while the available path sets at source node for each video session can be computed by the network server. With this information, the network server implements the proposed solution procedure and obtains the set of optimal paths and rate allocations for each video session. The network server then conveys this information to the source node of each video session. Source routing can be implemented on each path at a source node to ensure that the set of paths used strictly conform to the optimal solution. Network dynamics such as new session arrival, existing session departure, or major change in the link statistics would prompt the network server to recompute a new $(1-\epsilon)$-optimal solution. Consequently, this approach is effective only if the time scale for such network dynamics is much larger than the execution of the algorithm at the centralized server.

In the case when the time scale for network dynamics is small or too fast for the centralized server approach to be effective, the solution obtained in this paper is still significant, as it offers a theoretical performance benchmark for any proposed distributed algorithm and protocol for this problem.

\section{Simulation Results}

In this section, we present simulation results to illustrate the capability of the solution procedure. In each simulation, a wireless mesh network is generated by placing a number of nodes at random locations in a rectangular region. A wireless link exists if a node is within the transmission range of a node's radio. For 
TABLE II

Impact of VARying the Number of Paths Per Session FOR A 50-NODE NETWORK (CASE 1)

\begin{tabular}{ccc}
\hline $\begin{array}{c}\text { Paths per session } \\
(p p s)\end{array}$ & $\begin{array}{c}(1-\epsilon) \text {-optimal } \\
\text { Solution }\end{array}$ & $\begin{array}{c}\text { Computation Time } \\
(\mathrm{sec} .)\end{array}$ \\
\hline 1 & 168.03 & 0.89 \\
2 & 106.25 & 4.81 \\
3 & 101.3 & 9.12 \\
4 & 100.38 & 24.52 \\
5 & 98.56 & $>10 \mathrm{~min}$ \\
\hline
\end{tabular}

TABLE III

Impact of Varying the Number of Paths Per Session FOR A 50-NODE NETWORK (CASE 2)

\begin{tabular}{ccc}
\hline $\begin{array}{c}\text { Paths per session } \\
(p p s)\end{array}$ & $\begin{array}{c}(1-\epsilon) \text {-optimal } \\
\text { Solution }\end{array}$ & $\begin{array}{c}\text { Computation Time } \\
(\text { sec. })\end{array}$ \\
\hline 1 & 184.23 & 0.89 \\
2 & 128.77 & 1.82 \\
3 & 125.90 & 3.59 \\
4 & 123.30 & 7.19 \\
5 & 123.19 & 22.43 \\
\hline
\end{tabular}

each source-destination pair, a set of given paths are pre-computed using the $k$-shortest path routing algorithm [8].

In the simulations, each video session has a rate between $20 \mathrm{~Kb} / \mathrm{s}$ and $200 \mathrm{~Kb} / \mathrm{s}$. We use an H.263+ codec and the first 200 frames from the "Foreman" trace in the quarter common intermediate format (QCIF). The video is encoded at 12.5 frames/s and an intra rate of $1 / 9$. The rate-distortion parameters are obtained from [19]. Failure probabilities of the wireless links are chosen from a uniform distribution between [1\%, 5\%]; the bandwidth of a link is chosen from a uniform distribution between $[50,400] \mathrm{Kb} / \mathrm{s}$. The proposed solution procedure is implemented in C, and the LINDO API 3.0 is used in solving the LP relaxation problem $\ell$-PSRA. For every sub-problem in the branch-and-bound tree, the local search algorithm presented in Section III-E is used to obtain a feasible solution from the LP relaxation solution.

\section{A. Impact of Paths Per Session}

We first investigate how the average distortion is affected by the number of paths per session, henceforth denoted by $p p s$. In reality, different sessions may choose different number of paths for rate allocation. However, for simplicity, we use the same number of paths for all video sessions in this paper. A 50-node network is used in this simulation with three video sessions, and we compute the average distortion values by varying pps from 1 to 5. A decoding deadline of $0.2 \mathrm{~s}$ is chosen along with an $\epsilon$ value of 0.1 . The results are presented in Table II.

One would expect that the average distortion is a non-increasing function of pps, as more paths will increase the optimization search space. This is true. However there is a price to pay for a large number of pps. The larger the number of paths for each video session, the larger the size of the optimization problem, which results in an increased computation time (see Table II). In particular, in this table, where the number of paths is increased from two to three, the computation time is increased from $4.81 \mathrm{~s}$ to $9.12 \mathrm{~s}$, but with only a marginal reduction in average distortion. Increasing pps to five results in an even larger increase (exponential) in computation time (as compared with $p p s=2$ or $p p s=3$ ), but without a significant reduction in

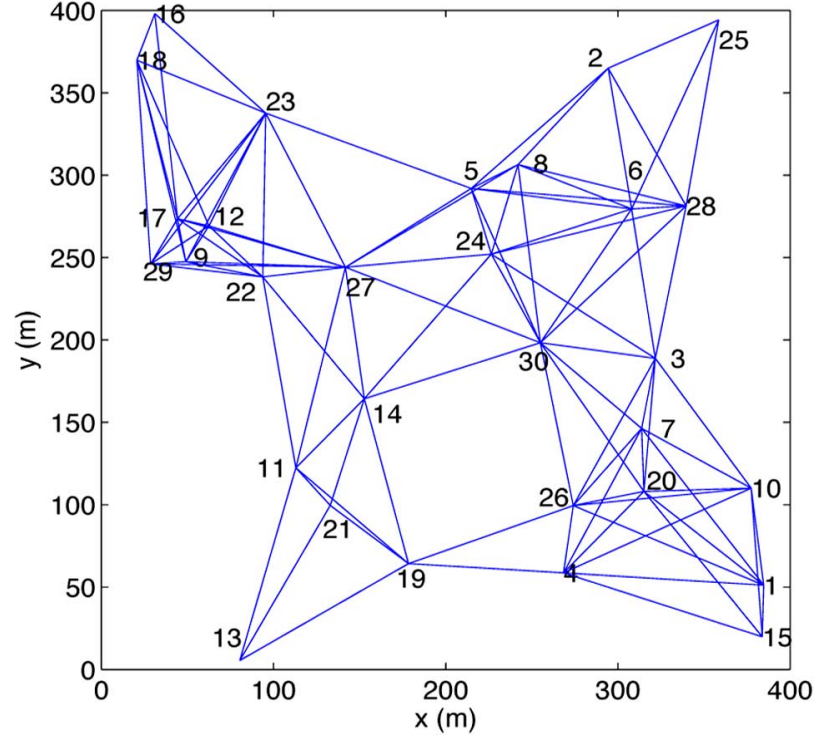

Fig. 3. A 30-node network for Cases I-III of Table IV.

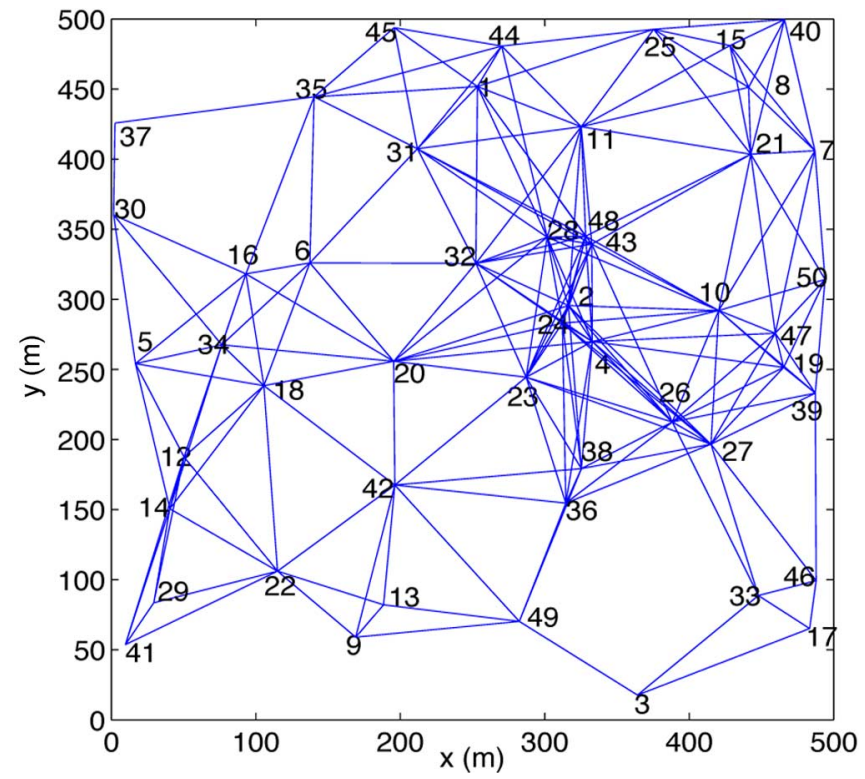

Fig. 4. A 50-node network for Cases IV-VIII of Table IV.

average distortion. As another instance, Table III presents the results for the same 50-node network but with a set of three different video sessions. Again, we can draw similar observation regarding the number of pps.

Therefore, a wise choice of pps is important in controlling computation time. In the rest of the simulation results, we use three paths for each video session.

\section{B. Performance of the Solution Procedure}

We next examine the performance of the proposed solution procedure for different problem instances, which are presented in Table IV. In the table, Cases I-III are for a 30-node network (Fig. 3) with one, two and three video sessions respectively. Cases III-VIII are for a 50-node network (Fig. 4), with one to five sessions, respectively. Based on the discussion from the last section, three paths have been used for each video session in 
TABLE IV

Performance of Our Solution Procedure for Different Problem InStances

\begin{tabular}{cccccc}
\hline Case & Network Size & No. of Sessions & Initial Feasible Solution & $(1-\epsilon)$-optimal Solution & $\epsilon$ \\
\hline I & 30 & 1 & 58.25 & 58.25 & 0.05 \\
II & 30 & 2 & 356.74 & 136.72 & 0.05 \\
III & 30 & 3 & 311.85 & 246.91 & 0.05 \\
IV & 50 & 1 & 88.01 & 48.25 & 0.1 \\
V & 50 & 2 & 120.49 & 82.18 & 0.1 \\
VI & 50 & 3 & 197.42 & 131.56 & 0.1 \\
VII & 50 & 4 & 224.10 & 206.49 & 0.1 \\
VIII & 50 & 5 & 255.09 & 239.27 & 0.1 \\
\hline
\end{tabular}

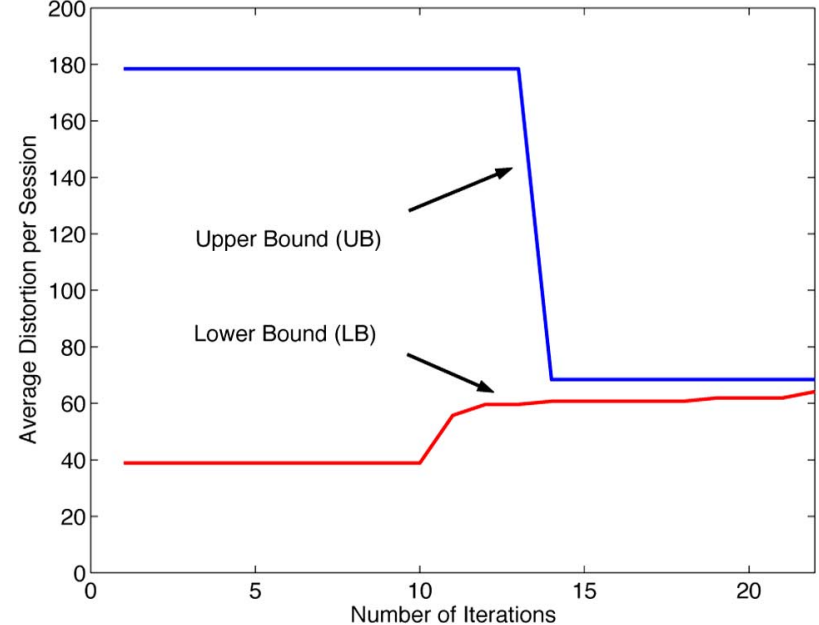

Fig. 5. Convergence of the solution for a 30-node network with two video sessions. (Case II).

all cases. The decoding deadline is chosen to be $0.1 \mathrm{~s}$ for all the cases. The fourth column of Table IV presents the total distortion values found via the corresponding UB for the original problem in the solution procedure (i.e., the UB obtained for the first problem of the branch-and-bound tree), and the fifth column of Table IV presents total distortion values for the $(1-\epsilon)$-optimal solutions.

In order to study the iterative convergence performance of the proposed solution procedure, we plot the evolution of the upper bound UB and the lower bound LB for a few cases from Table IV. Fig. 5 presents the results for the 30-node network with two video sessions. For this particular instance, where $\epsilon=$ $5 \%$, the optimality gap converges to a value of 6.74 with an upper bound value of UB $=136.72$ at the 22 nd iteration. Recall that at each iteration, the lower and upper bounds for the original problem are chosen according to (25). We observe that even though the initial gap between the lower and upper bounds is high, the gap closes within $\epsilon$ over the iterations. Similar observations can be made for all the other cases in Figs. 6-9.

\section{Impact of Decoding Deadline}

In Fig. 10, we plot the average distortion versus decoding deadline for the same 50-node network with two and three video sessions, respectively, each with three paths per session. For each point, we use the same decoding deadline value for all sessions. We observe that the average distortion is a non-increasing function of decoding deadline. For small decoding deadline values, most of the video packets are overdue,

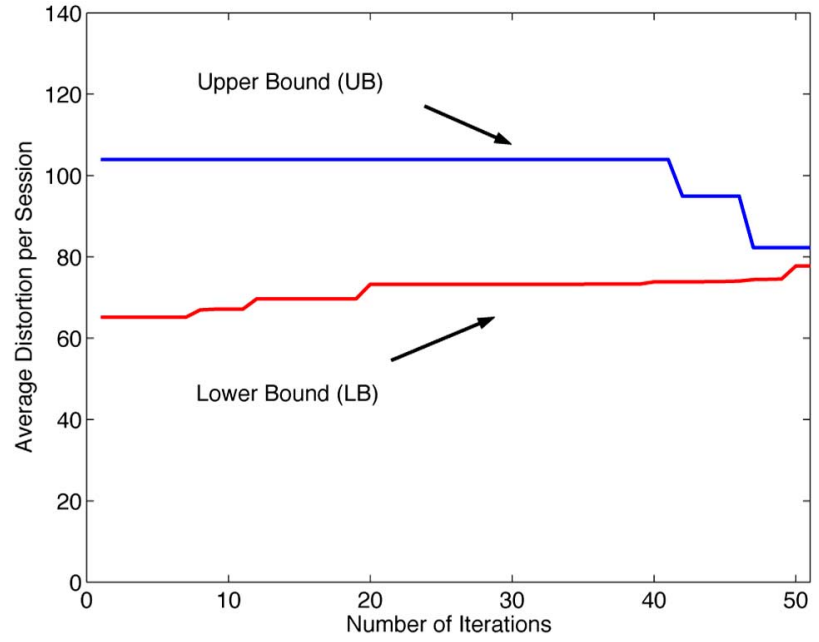

Fig. 6. Convergence of the solution for a 30-node network with three video sessions. (Case III).

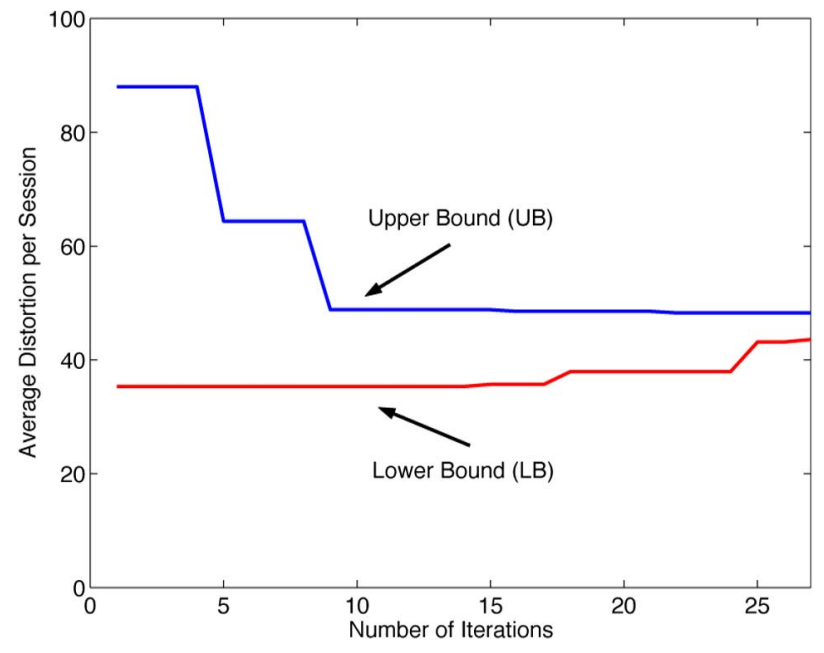

Fig. 7. Convergence of the solution for a 50-node network with one video sessions. (Case IV).

resulting in high distortion. As decoding deadline increases, the average distortion quickly decreases because more and more packets are now received in time, contributing to an improved video quality. As the decoding deadline further increases, fewer and fewer packets are overdue and the reduction in distortion becomes negligible.

\section{RELATED WORK}

For video communications, several path selection schemes have been developed for various network settings (e.g., [1], 


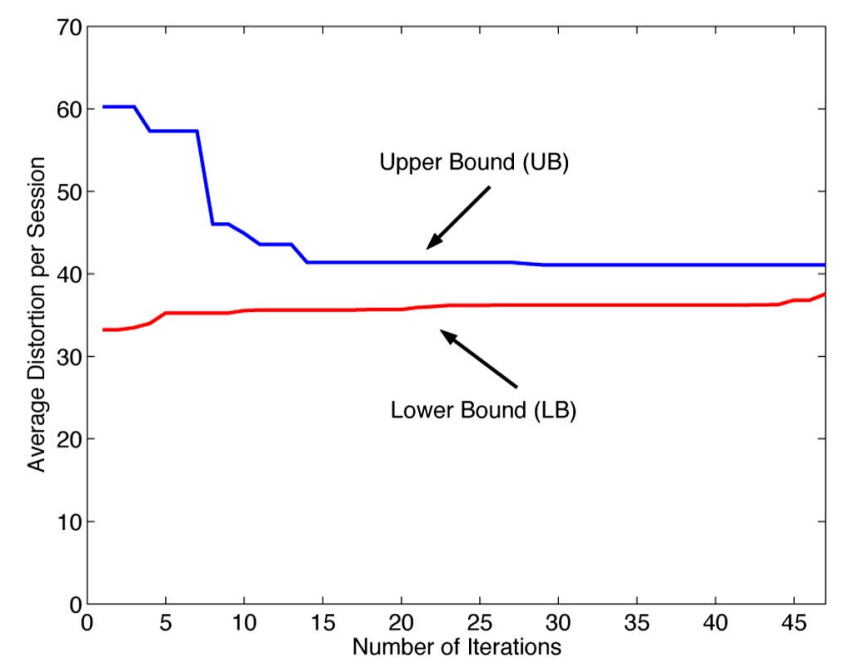

Fig. 8. Convergence of the solution for a 50-node network with two video sessions. (Case V).

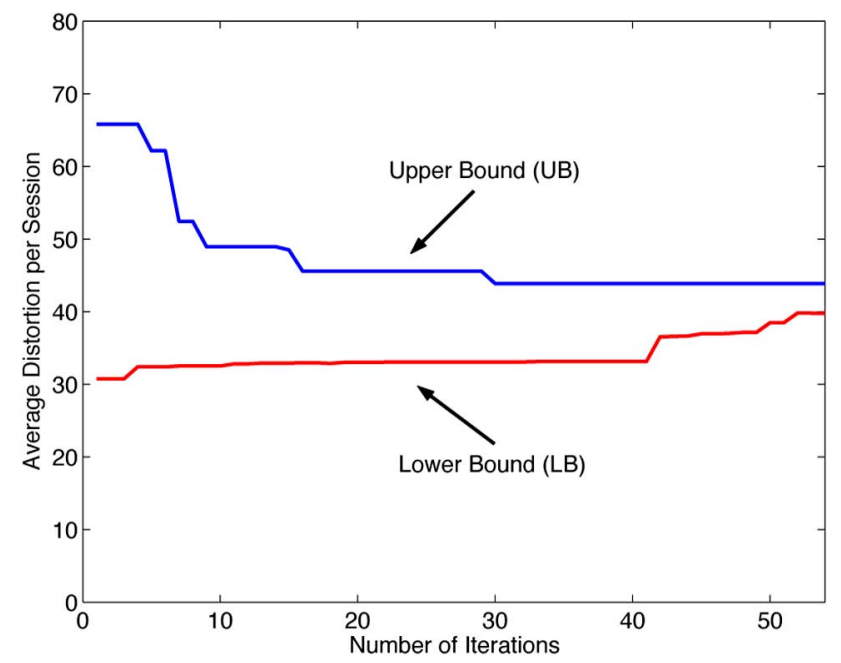

Fig. 9. Convergence of the solution for a 50-node network with three video sessions. (Case VI).

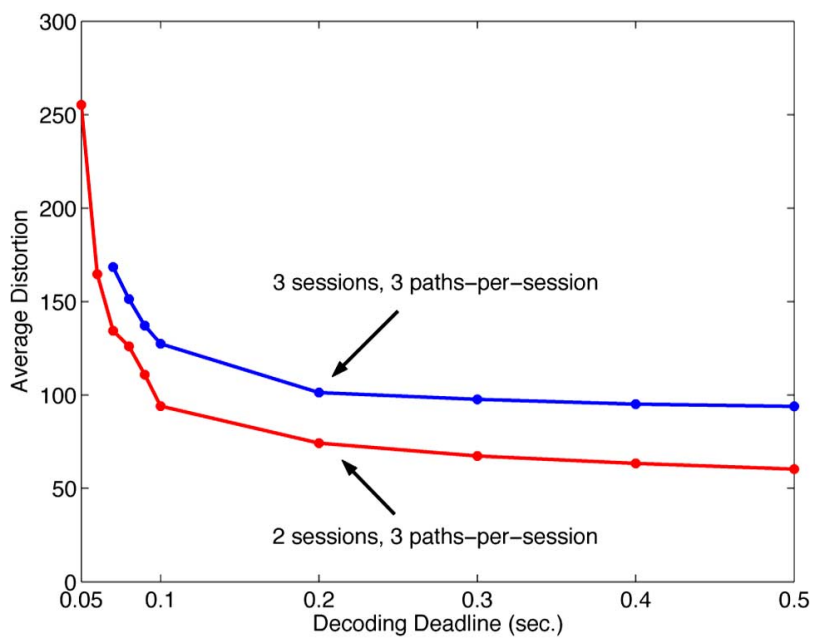

Fig. 10. Average distortion versus decoding deadline for a 50-node network.

[4], [11], [12], [20]). In [1], Apostolopoulos et al. showed that multiple description (MD) video streaming provides an effective means of exploiting the path diversity provided by a Content Delivery Network (CDN) infrastructure. They proposed three heuristic schemes for selecting a pair of MD video servers in a CDN framework, and showed that the combination of MD coding and path diversity offers better error-resilience for streaming media. In a more recent work [20], Wei et al. proposed a model to estimate the concurrent packet drop probability of a node-disjoint path pair, and used this metric to compare different path pairs for video streaming in a wireless ad hoc network. In their problem formulation, paths are selected by identifying a pair of node-disjoint paths that minimizes the concurrent packet drop probability over all possible path pairs. Since this problem formulation is NP-hard, they proposed a heuristic solution to find each path in the pair based on its likelihood of minimizing the combined packet drop probability.

In [4], Begen et al. studied the problem of selecting a pair of paths that maximizes the average video quality (minimize the end-to-end distortion) for MD video streaming in service overlay networks. They proposed to use brute-force exhaustive search to find an optimal path pair over all possible combinations. They also proposed a heuristic-based solution for the optimal multi-path selection problem in [3]. Apart from heuristics, the model for MD video in [3] and [4] did not take into account the impact of latency in computing the end-to-end video distortion. Such latency consideration is important for real-time video, although it will increase problem complexity in the formulation, as we have seen in this paper.

In [11], Mao et al. studied optimal routing for point-to-point MD video communications in a wireless ad hoc network. The focus there was on cross-layer optimal routing and the problem was solved using a metaheuristic algorithm instead of rigorous optimization solution as in this paper. The problem of optimal routing for multiple video sessions was studied in [12], where again a metaheuristic algorithm was used.

Finally, Setton et al. presented a multi-path congestion-based traffic partitioning scheme in [16] for optimizing received video quality in bandwidth-limited wireless ad hoc networks. By focusing on only a single video session and thereby ignoring the interaction among multiple sessions, the authors were successful in formulating the flow assignment problem as a convex optimization problem. However, in the presence of multiple concurrent sessions, modeling the interaction among these sessions increases the problem complexity considerably, as in the case of our OPT-PSRA problem.

\section{CONCLUSION}

In this paper, we investigated the joint problem of path selection and rate allocation for concurrent video sessions in a wireless mesh network. We formulated this problem as a non-convex optimization problem and developed a solution procedure based on branch-and-bound framework and reformulation-linearization technique. This solution approach is shown to produce provably $(1-\epsilon)$-optimal solutions. This result is significant as it not only provides theoretical understanding on this cross-layer optimization problem, but also offers a performance benchmark for any future proposed distributed algorithm and protocol for this problem. We also used simulation results to demonstrate the efficacy of the solution procedure for various network instances. 


\section{REFERENCES}

[1] J. G. Apostolopoulos, T. Wong, W. Tan, and S. Wee, "On multiple description streaming in content delivery networks," in Proc. IEEE INFOCOM, New York, Jun. 2002, pp. 1736-1745.

[2] Handbook of Evolutionary Computation, T. Back, D. Fogel, and Z. Michalewicz, Eds. New York: Oxford Univ. Press, 1997.

[3] A. C. Begen, Y. Altunbasak, and O. Ergun, "Fast heuristics for multipath selection for multiple description encoded video streaming," in Proc. IEEE ICME, Jul. 2003, pp. 517-520.

[4] A. C. Begen, Y. Altunbasak, and O. Ergun, "Multi-path selection for multiple description encoded video streaming," EURASIP Signal Process.: Image Commun., vol. 20, no. 1, pp. 39-60, Jan. 2005.

[5] H. Chernoff, "A measure of asymptotic efficiency for tests of a hypothesis based on the sum of observations," Ann. Math. Statist., vol. 23, pp. 493-507, 1952.

[6] Z. Duan, Z.-L. Zhang, Y. T. Hou, and L. Gao, "A core stateless bandwidth broker architecture for scalable support of guaranteed services," IEEE Trans. Parallel Distrib. Syst., vol. 15, no. 2, pp. 167-182, Feb. 2004.

[7] A. Elwalid, D. Heyman, T. V. Lakshman, D. Mitra, and A. Weiss, "Fundamental bounds and approximations for ATM multiplexers with applications to video teleconferencing," IEEE J. Sel. Areas Commun., vol. 13 , no. 6, pp. 953-962, Aug. 1995.

[8] D. Eppstein, "Finding the $k$ shortest paths," SIAM J. Comput., vol. 28, no. 2, pp. 652-673, Aug. 1999.

[9] M. R. Garey and D. S. Johnson, Computers and Intractability: A Guide to the Theory of NP-Completeness. New York: W. H. Freeman \& Co., 1979.

[10] T. Kuang and C. Williamson, "A measurement study of RealMedia audio/video streaming traffic," in Proc. SPIE ITCOM 2002, Boston, MA, Jul. 2002, pp. 68-79.

[11] S. Mao, Y. T. Hou, X. Cheng, H. D. Sherali, and S. F. Midkiff, "Multi-path routing for multiple description video over wireless ad hoc networks," in Proc. IEEE INFOCOM, Miami, FL, Mar. 2005, pp. $740-750$.

[12] S. Mao, S. Kompella, Y. T. Hou, H. D. Sherali, and S. F. Midkiff, "Routing for multiple concurrent video sessions in wireless ad hoc networks," in Proc. IEEE ICC, Seoul, Korea, May 2005, pp. 1229-1235.

[13] S. Murthy and J. J. Garcia-Luna-Aceves, "Congestion-oriented shortest multi-path routing," in Proc. IEEE INFOCOM, San Francisco, CA, May 1996, pp. 1038-1036.

[14] I. Norros, "On the use of fractional Brownian motion in the theory of connectionless networks," IEEE J. Sel. Areas Commun., vol. 13, no. 6, pp. 953-962, Aug. 1995 .

[15] P. Papadimitratos, Z. J. Haas, and E. G. Sirer, "Path set selection in mobile ad hoc networks," in Proc. ACM MobiHoc, Lausanne, Switzerland, Jun. 2002, pp. 1-11.

[16] E. Setton, X. Zhu, and B. Girod, "Congestion-optimized multi-path streaming of video over ad hoc wireless networks," in Proc. IEEE ICME, Taipei, Taiwan, Jun. 2004, pp. 1619-1622.

[17] H. D. Sherali and C. H. Tuncbilek, "A global optimization algorithm for polynomial programming problems using a reformulation-linearization technique," J. Global Optim., vol. 2, no. 1, pp. 101-112, 1992.

[18] H. D. Sherali and W. P. Adams, A Reformulation-Linearization Technique for Solving Discrete and Continuous Nonconvex Problems. Boston, MA: Kluwer Academic, 1999.

[19] K. Stuhlmuller, N. Farberand, M. Link, and B. Girod, "Analysis of video transmission over lossy channels," IEEE J. Sel. Areas Commun., vol. 18 , no. 6, pp. 1012-1032, Jun. 2000.

[20] W. Wei and A. Zakhor, "Path selection for multi-path streaming in wireless ad hoc networks," in Proc. IEEE ICIP, Atlanta, GA, Oct. 2006, pp. 3045-3048.

[21] Z.-L. Zhang, Z. Duan, and Y. T. Hou, "On scalable design of bandwidth brokers," IEICE Trans. Commun., vol. E84-B, no. 8, pp. 2011-2025, Aug. 2001.

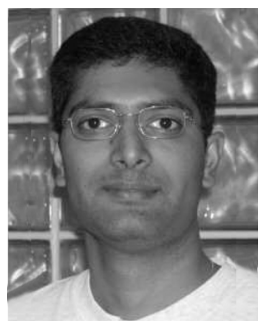

Sastry Kompella (S'04-M'07) received the B.E. degree in electronics and communication engineering from Andhra University, India, in May 1996, the M.S. degree in electrical engineering from Texas Tech University, Lubbock, TX, in May 1998, and the Ph.D. degree in electrical and computer engineering from Virginia Polytechnic Institute and State University, Blacksburg, VA, in 2006.

Currently, he is a Researcher at the U.S. Naval Research Laboratory, Information Technology Division, Washington, DC. His research focuses on complex problems in cross-layer optimizations and multimedia delivery over wireless networks.

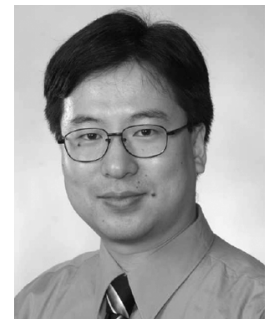

Shiwen Mao (S'99-M'04) received the B.S. and M.S. degrees from Tsinghua University, Beijing, China, in 1994 and 1997, respectively, both in electrical engineering. He received the M.S. degree in system engineering and the $\mathrm{Ph} . \mathrm{D}$. degree in electrical and computer engineering from Polytechnic University, Brooklyn, NY, in 2000 and 2004, respectively.

He was a Research Member with the IBM China Research Lab, Beijing, from 1997 to 1998, and a research intern at Avaya Labs-Research, Holmdel, $\mathrm{NJ}$, in the summer of 2001. He was a Research Scientist in the Bradley Department of Electrical and Computer Engineering, Virginia Tech, Blacksburg, VA, from December 2003 to April 2006. Currently, he is an Assistant Professor in the Department of Electrical and Computer Engineering, Auburn University, Auburn, AL. He is the coauthor of a textbook, TCP/IP Essentials: A Lab-Based Approach (Cambridge University Press, 2004). His research interests include cross-layer design and optimization of wireless networks, cognitive networks, and multimedia communications.

Dr. Mao is on the Editorial Board of the Hindawi Advances in Multimedia Journal and the Wiley International Journal of Communication Systems. He received the 2004 IEEE Communications Society Leonard G. Abraham Prize in the Field of Communications Systems. He also received a Certificate of Appreciation from the IEEE Computer Society in 2007. His current research is supported in part by the National Science Foundation under Grant ECCS-0802113 and through the Wireless Internet Center for Advanced Technology (WICAT).

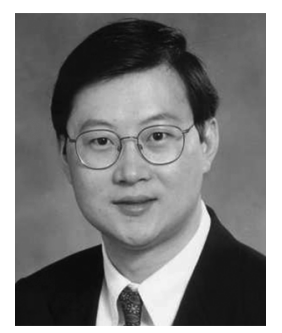

Y. Thomas Hou (S'91-M'98-SM'04) received the B.E. degree from the City College of New York, New York, NY, in 1991, the M.S. degree from Columbia University, New York, NY, in 1993, and the Ph.D. degree from Polytechnic University, Brooklyn, NY, in 1998, all in electrical engineering.

Since August 2002, he has been with the Bradley Department of Electrical and Computer Engineering, Virginia Polytechnic Institute and State University (Virginia Tech), Blacksburg, VA, where he is now an Associate Professor. His current research interests are radio resource (spectrum) management and networking for cognitive radio wireless networks, optimization and algorithm design for wireless ad hoc and sensor networks, and video communications over dynamic ad hoc networks From 1997 to 2002, he was a Researcher at Fujitsu Laboratories of America, Sunnyvale, CA, where he worked on scalable architectures, protocols, and implementations for differentiated services Internet, service overlay networking, video streaming, network bandwidth allocation policies, and distributed flow control algorithms.

Dr. Hou received an Office of Naval Research (ONR) Young Investigator Award in 2003 and a National Science Foundation (NSF) CAREER Award in 2004 for his research on optimizations and algorithm design for wireless ad hoc and sensor networks. He has published extensively in leading journals and top-tier conferences, and has received five best paper awards from IEEE (including IEEE INFOCOM 2008 Best Paper Award and IEEE ICNP 2002 Best Paper Award). He holds five U.S. patents. He is active in professional services and is currently serving as an Editor of IEEE TRANSACTIONS ON WIRELESS COMMUNICATIONS, ACM/Springer Wireless Networks (WINET), and Elsevier Ad Hoc Networks. He is a past Associate Editor of IEEE TRANSACTIONS on Vehicular Technology. He was Co-Chair of the Technical Program Committee (TPC) of the Second International Conference on Cognitive Radio Oriented Wireless Networks and Communications (CROWNCOM 2007). He was the Founding Chair of the First IEEE Workshop on Networking Technologies for Software Defined Radio Networks, 2006. He was Co-Chair (with T. Ephremides) of the NSF Workshop on Bridging the Gap between Wireless Networking Technologies and Advances at the Physical Layer, 2007. $\mathrm{He}$ is TPC Co-Chair of IEEE INFOCOM 2009. 


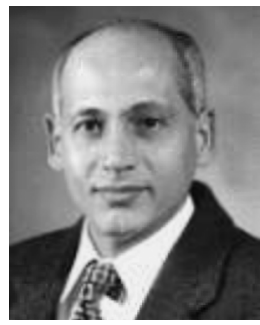

Hanif D. Sherali received the B.S. degree in electrical engineering from Bombay University, India, in 1975, the M.S. and Ph.D. degrees in operations research from the Georgia Institute of Technology, Atlanta, in 1977 and 1979, respectively.

He is a University Distinguished Professor and the W. Thomas Rice Chaired Professor of Engineering in the Industrial and Systems Engineering Department at Virginia Polytechnic Institute and State University (Virginia Tech), Blacksburg, VA. His areas of research interest are in analyzing problems and designing algorithms for specially structured linear, nonlinear, and integer programs arising in various applications, global optimization methods for non-convex programming problems, location and transportation theory and applications, economic and energy mathematical modeling and analysis. $\mathrm{He}$ has published over 238 refereed articles in various operations research journals, has authored or co-authored six books in this area, and serves on the editorial board of eight journals.

Dr. Sherali is an elected member of the U.S. National Academy of Engineering. 